\title{
REVIEW
}

\section{Incidence and prevalence of idiopathic pulmonary fibrosis: review of the literature}

\author{
Luba Nalysnyk*, Javier Cid-Ruzafa\#, Philip Rotella* and Dirk Esser"
}

ABSTRACT: Idiopathic pulmonary fibrosis (IPF) is a chronic, progressive, fibrosing interstitial pneumonia of unknown aetiology. It is a rare disease, and its incidence and prevalence are not clear. Therefore, we sought to review the published evidence on the global epidemiology of IPF.

A comprehensive review of English language literature was performed by searching Medline and EMBASE for studies on IPF epidemiology published between January 1990 and August 2011. Studies providing quantitative data on IPF incidence and/or prevalence were identified and key data collected.

15 studies reporting on the incidence and/or prevalence of IPF were identified and summarised. IPF prevalence estimates in the USA varied between 14 and 27.9 cases per 100,000 population using narrow case definitions, and 42.7 and 63 per 100,000 population using broad case definitions. In Europe, IPF prevalence ranged from 1.25 to 23.4 cases per 100,000 population. The annual incidence of IPF in the USA was estimated at $6.8-8.8$ per 100,000 population using narrow case definitions and 16.3-17.4 per 100,000 population using broad case definitions. In Europe, the annual incidence ranged between 0.22 and 7.4 per 100,000 population. IPF prevalence and incidence increase with age, are higher among males and appear to be on the increase in recent years.

IPF is an orphan disease that affects a potentially increasing number of people in Europe and the USA. The observed variability in IPF incidence and prevalence may be explained by the differences in diagnostic criteria used, case definition, study population and study design.

KEYWORDS: Cryptogenic fibrosing alveolitis, epidemiology, interstitial lung disease, usual interstitial pneumonia

diopa1thic pulmonary fibrosis (IPF) is defined by the main thoracic and respiratory professional societies as "a specific form of chronic, progressive, fibrosing interstitial pneumonia of unknown cause, occurring primarily in older adults, limited to the lungs, and associated with the histopathologic and/or radiologic pattern of usual interstitial pneumonia (UIP)" [1]. IPF usually manifests with breathlessness on exertion, increasing cough and dyspnoea, bibasilar inspiratory crackles and worsening pulmonary function tests, as well as finger clubbing in $\sim 50 \%$ of patients [1-3]. Respiratory decline can be slow and progressive (the most frequent clinical phenotype), with or without acute exacerbations, or rapid and accelerated, resulting in distinct patterns of survival [1-4]. Diagnosis is usually made 6 to 24 months after the initial symptoms $[3,5]$.
IPF is the most common and severe form of idiopathic interstitial pneumonias (IIPs), it is irreversible, has an unpredictable and variable clinical course and is associated with extremely poor prognosis [1-3]. In 2000, the American Thoracic Society (ATS) collaborated with the European Respiratory Society (ERS) and developed a consensus statement for the diagnosis and treatment of IPF [6], which was further revised in 2011 [1]. According to the updated guidelines, an IPF diagnosis requires the exclusion of known causes of interstitial lung disease (ILD) and either the presence of a typical UIP pattern on highresolution computed tomography (HRCT) scans or a specific lung biopsy pattern.

The incidence and prevalence of IPF varies across studies. Recent studies indicate that the incidence

\section{AFFILIATIONS}

*United BioSource Corporation, Lexington, MA, USA.

\#United BioSource Corporation, London, UK.

"Boehringer Ingelheim GmbH, Ingelheim, Germany.

CORRESPONDENCE

L. Nalysnyk

Genzyme Corporation

500 Kendall Street

Cambridge

MA 02142

USA

E-mail: Inalysnyk@aol.com

Received:

March 262012

Accepted after revision:

April 132012

PROVENANCE

Publication of this peer-reviewed article was supported by Boehringer Ingelheim, Germany (article sponsor European Respiratory Review issue 126) 
and mortality of IPF are on the increase $[7,8]$. However, the true incidence and prevalence of IPF are not well established due to the lack of a uniform definition of IPF in older studies, diagnostic criteria, differences in case finding methodologies and study designs.

Recent publications have reviewed the currently accepted pathogenesis of IPF, as well as its diagnostic and therapeutic recommendations [1-3]. Our goal was to review and summarise the published evidence on the global prevalence and incidence of IPF.

\section{METHODS}

\section{Literature search and study identification}

A comprehensive literature review was performed by searching the Medline (via PubMed) and EMBASE databases for relevant studies on IPF epidemiology published from January 1990 to August 2011.

Combinations of search terms used included "idiopathic pulmonary fibrosis", "idiopathic interstitial pneumonias", "cryptogenic fibrosing alveolitis", "usual interstitial pneumonia", "fibrosing alveolitis", "epidemiology", "incidence" and "prevalence". Searches were limited to original research studies of humans. Reference lists of key articles were manually reviewed to supplement the electronic searches and ensure that the most relevant studies were identified.

Study selection was accomplished through two levels of study screening. At the abstract level, the following publications were excluded: case reports, letters, commentaries, editorials, reviews, animal and in vitro studies, studies on IPF pathophysiology or aetiology, diagnostic studies and clinical trials. The most relevant English language articles of accepted abstracts were retrieved for further review. Observational studies reporting on IPF incidence and/or prevalence were included for this review. Two reviewers had to agree on each accepted and rejected study. From the articles reviewed, we identified studies providing quantitative evidence of the incidence and/or prevalence of IPF. Age- and sex-stratified IPF estimates, as well as trends over time, were captured if reported.

\section{RESULTS}

The literature search through Medline (via PubMed) and EMBASE yielded 1,676 citations, after excluding duplicate citations from the various sources. At the abstract level, 1,470 abstracts were rejected. The full publications of 224 English language articles, including recent reviews, were retrieved for further review, and a total of 15 English language studies [7-21] are summarised in this article. 12 studies reported IPF prevalence [9-20] and 11 studies reported IPF incidence [7-9, $11,13,14,16-18,20,21]$, with eight studies reporting data for both prevalence and incidence of IPF $[9,11,13,14,16-18,20]$.

\section{Prevalence of IPF}

We identified 12 studies that reported prevalence of IPF among various populations. Prevalence studies were identified for countries in North America (USA n=3), Europe (Belgium $n=1$, Czech Republic $n=1$, Finland $n=1$, Greece $n=1$, Italy $n=1$, Norway $\mathrm{n}=1$, multinational $\mathrm{n}=2$ ) and Asia (Japan $\mathrm{n}=1$ ). Furthermore, four studies reported prevalence stratified by age, three stratified by sex, three stratified by disease subtype, two stratified by case definition, one stratified by study period, one stratified by geographic region, and one compared prevalence between countries. Prevalence was usually expressed as the proportion of the population with an existing diagnosis of IPF and reported per 100,000 population. In a few cases, IPF prevalence was reported as the proportion of patients among those with ILD. Of the 12 prevalence studies, 10 reported IPF prevalence among general populations and two reported IPF prevalence among ILD patients. Table 1 summarises the prevalence estimates among general populations reported per 100,000 population by geographic region.

In the USA, the prevalence of IPF varied depending on the population studied and the case definition used to identify IPF patients: from 14 to 27.9 cases per 100,000 of the general population using narrow case definitions and from 42.7 to 63 cases per 100,000 population using broad case definitions. FERNÁNDEZ-PÉREZ et al. [11] conducted a population-based study in Olmsted County, MN, USA, for the identification of IPF through a medical record linkage system. The authors used two case definitions: the narrow case definition had to meet all major and minor ATS/ERS criteria [22] and required definite UIP patterns on HRCT scans, while the broad case definition included the patients meeting the narrow definition, as well as those with HRCT features characterised as possible UIP. The age- and sex-adjusted prevalence estimates in people $\geqslant 50 \mathrm{yrs}$ of age were 27.9 cases and 63.0 cases per 100,000 population using a narrow and a broad case definition, respectively. RAGHU et al. [16] also applied two definitions to a healthcare claims processing system and, using US census data, estimated the IPF prevalence in the USA to be 14.0 based on their narrow definition and 42.7 per 100,000 population based on their broad definition. These two US studies demonstrate how varying case definitions within the same study population can significantly impact prevalence estimates of IPF.

In European countries, IPF prevalence ranged from 1.25 per 100,000 population in Belgium [17] to 23.4 per 100,000 population in Norway [20]. THOMEER et al. [17] set up a prospective registry where respiratory specialists from 20 centres in Flanders (Belgium) registered their ILD patients; IPF cases required biopsy with UIP. VON PLESSEN et al. [20] used discharge diagnoses from a single centre to identify candidate patients, who were then defined as having IPF by exclusion of other conditions where pathogenesis could be explained. Other authors used different methodologies (or did not detail case identification) and reported prevalence estimates of intermediate value ( 3.38 to $16-18$ cases per 100,000 population) [12-14]. KOLEK [14] did not give a case definition at the time of his retrospective study, but reported increasing IPF prevalence in the study period between 1981 and 1990 in the Czech Republic from 6.5 to 12.1 cases per 100,000 population. In Finland, HoDGSON et al. [12] screened hospital databases nationwide for alveolitis fibroticans idiopathica and used the international consensus criteria published by the ATS and ERS in 2000 [6]; the nationwide prevalence of IPF for the period 1997-1998 ranged from 16 to 18 cases per 100,000 population. KARAKATSANI et al. [13] conducted a national survey of pulmonologists in Greece with a special interest in ILD and relied on the expert evaluations of the corresponding pathologist and radiologist, using the same process and diagnostic algorithm in all centres; the estimated IPF prevalence in 2004 was 3.38 per 100,000 


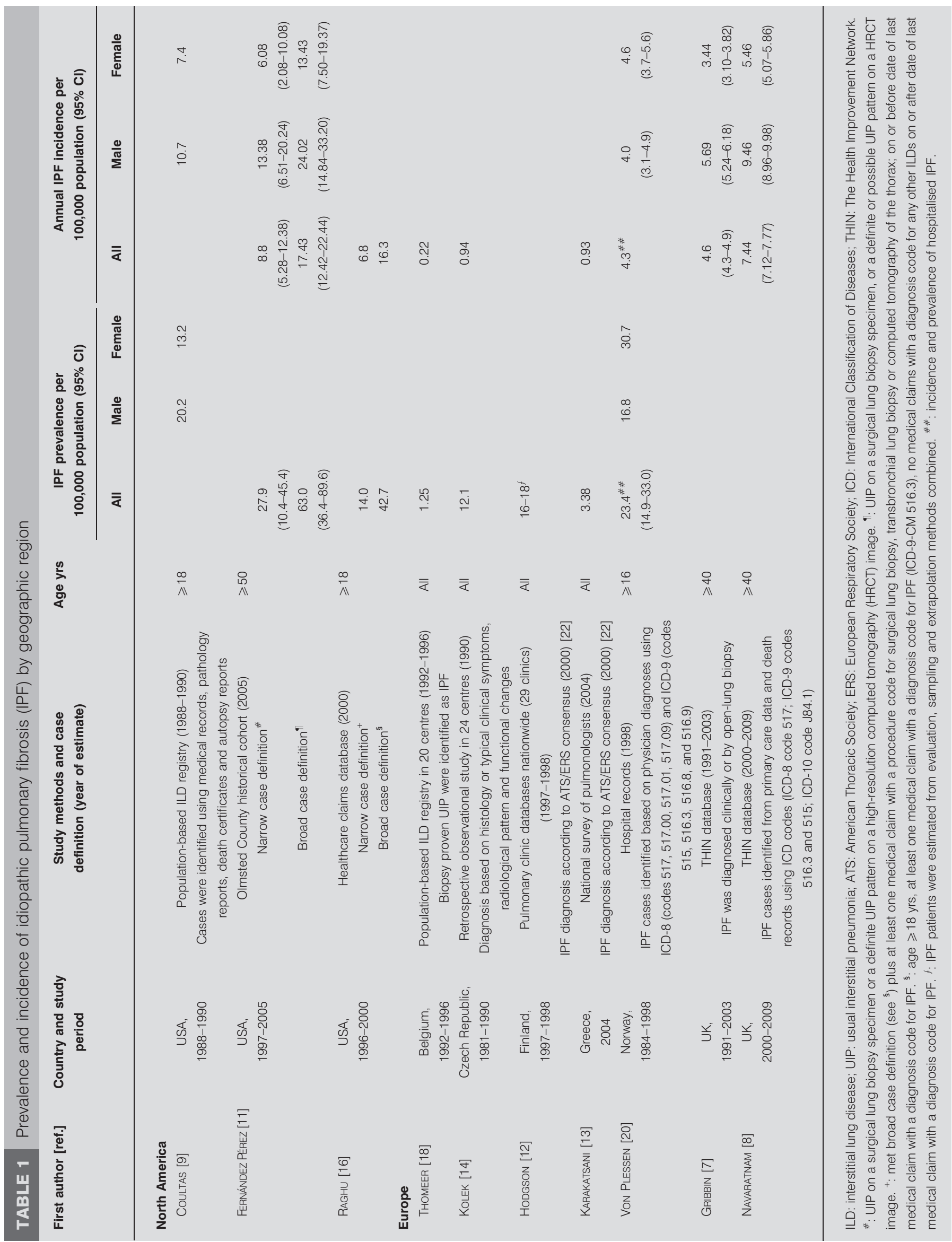


population. In general, prevalence estimates appear to be lower in Europe relative to those reported in the US populations.

A study from Japan did not directly report the prevalence of IPF, although the data was used to calculate approximate estimates. OHNO et al. [15] used personal clinical records of patients with a certificate of medical benefits for IIP and applied the 2003 Japanese adaptation of the 2002 international guidelines [22] to classify cases as IPF. The annual prevalence of IIP was 3.44 per 100,000 population. Clinical records were available for $35.1 \%$ of IIP cases; of these, $85.7 \%$ were classified as IPF. Assuming the same proportion of IPF in those IIP cases that had no clinical records, one could estimate an overall IPF prevalence of 2.95 per 100,000 population. This figure may underestimate the true prevalence because only the highest severity cases are granted benefits; however, it may be an overestimate because most patients were diagnosed based on clinical, rather than pathological, findings.

Another study focused on IIP in a paediatric population: DINWIDDIE et al. [10] conducted a survey among paediatricians known to have an interest in paediatric pulmonology in the UK and Ireland. In order to be included in the study, patients had to have had a lung biopsy. During a 3-yr period, a total of 46 ILD cases were identified, of which 10 were diagnosed with fibrosing alveolitis and another 18 with mixed fibrosing alveolitis/desquamative interstitial pneumonitis (DIP). The estimated prevalence rate of ILD in the UK and Ireland for children aged 0-16 yrs was reported to be 3.6 cases per million. Assuming that cases with fibrosing alveolitis (either with or without DIP) are indicative of IPF, one could estimate the IPF prevalence in children to be $0.78-2.2$ cases per million.

The difference in case definition and identification methods precludes a specific comparison of prevalence estimates among different countries.

\section{IPF prevalence by age, sex and trend over time}

Three studies reported IPF prevalence by sex and age $[9,16$, 20]. In the USA, CoulTAS et al. [9] reported that males had higher prevalence of IPF than females, and RAGHU et al. [16] reported higher IPF prevalence among older males ( $\geqslant 65$ yrs) relative to younger males in their study using a large healthcare claims database. In Europe, a similar trend of increasing prevalence with increasing age was seen in a study from Norway [20]; however, females seemed to have higher IPF prevalence than males (fig. 1). One study reported on the trend in IPF prevalence over time; KOLEK [14] evaluated the epidemiology of IPF in the Czech Republic between 1981 and 1990 and found that the prevalence increased from 6.5 to 12.1 per 100,000 population over the 10 -yr period. In general, IPF prevalence increases with age, with the highest prevalence observed in patients $\geqslant 75$ yrs of age, has no clear sex trend and appears to be on the rise in Europe.

\section{Proportion of IPF among subjects with ILD}

In their review of published studies of ILD registries, THOMEER et al. [18] compared the distribution of different ILD diagnoses across two European countries (Belgium and Italy) and the USA. In the US prospective registry in Bernalillo County, NM [9], $23 \%$ of ILD subjects were diagnosed with IPF. The lowest IPF proportion among ILD subjects was reported in Belgium

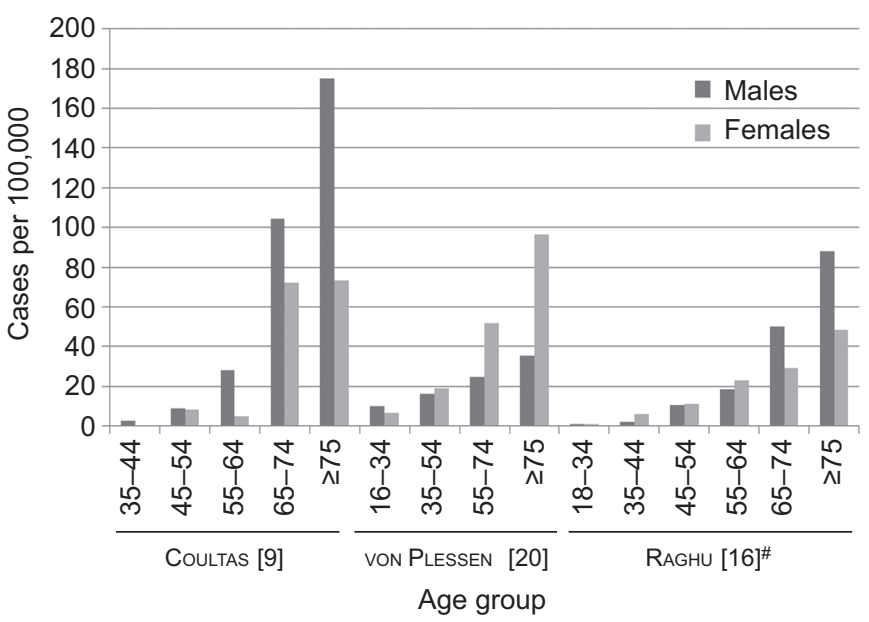

FIGURE 1. Age- and sex-stratified prevalence of idiopathic pulmonary fibrosis. \# : narrow case definition.

(Flanders, 17\%). Similar results were found in an Italian retrospective registry (19\% during 1978-1998); however, in a prospective Italian Registry of Diffuse Infiltrative Pulmonary Diseases (RIPID), the IPF fraction was higher (37\% during 1997-1999). TINELLI et al. [19] analysed results from the RIPID registry after it changed from fax-based enrolment (RIPID-1) to a more efficient web-based enrolment modality (RIPID-2) and reported that, from late 2000 to early 2005, IPF represented $27.4 \%$ of ILD diagnoses (95\% CI 25.8-29.0). Based on these data, IPF accounts for $17-37 \%$ of all ILD diagnoses.

\section{Incidence of IPF}

The literature search identified a total of 11 studies published between 1990 and 2011 reporting incidence of IPF among various populations. Incidence studies were identified for countries in North America (USA $n=3$ ) and Europe (Belgium $n=1$, Czech Republic $n=1$, Greece $n=1$, Norway $n=1$, Spain $n=1$, UK $n=2$, multinational $n=1$ ). Nine studies presented incidence rates per 100,000 population per yr and two studies reported incidence cases as proportions of ILD cases. Annual IPF incidence estimates per 100,000 population by geographic region are summarised in table 1.

In the USA, RAGHU et al. [16] extrapolated their observed rates to the US adult population and estimated the annual incidence of IPF to be 6.8 per 100,000 population with a narrow IPF definition and 16.3 per 100,000 population with a broad IPF definition. Similarly, FERNÁNDEZ PÉREZ et al. [11] reported that age- and sexadjusted incidence among residents $\geqslant 50$ yrs of age from 1997 to 2005 ranged from 8.8 per 100,000 person-yrs with a narrow case definition to 17.4 per 100,000 person-yrs with a broad case definition.

In Europe, the annual IPF incidence ranged from 0.22 per 100,000 population in Belgium [17] to 7.94 per 100,000 population in the UK [8]. Navaratnam et al. [8] used The Health Improvement Network (THIN), a computerised longitudinal primary care database recorded by UK general practitioners as part of routine clinical care, to define IPF clinical syndrome with specific Read codes, excluding coexisting diagnoses of connective tissue disease, extrinsic allergic alveolitis, sarcoidosis, 
pneumoconiosis or asbestosis. GRIBBIN et al. [7] also utilised THIN but applied a more specific case definition with fewer Read codes. Using this approach, the crude annual incidence of IPF was estimated to be 4.6 per 100,000 population. The annual incidence of IPF was similar in Greece (0.93 per 100,000 population in 2004) [13] and the Czech Republic (1.28 per 100,000 population in 1990 , mean 0.94 per 100,000 population during 1981-1990), where it was highest in the regions of the four major diagnostic centres in 1990 (1.58 per 100,000 population) and lowest in vinicultural areas (0.59 per 100,000 population). The authors concluded that diagnostic capabilities dominate over ecological or environmental factors in determining IPF detection rates [14].

No studies of IPF incidence in geographic regions other than the USA or Europe were identified. Similar to IPF prevalence estimates, annual incidence rates appear to be higher in the USA relative to those reported in European countries. However, these findings cannot be compared directly between countries due to the differences in case finding methodology.

\section{IPF incidence by age, sex and trend over time}

In the USA, males had higher incidence than females (10.7 versus 7.4 per 100,000 person-yrs) [9]. In Europe, a similar trend of increasing IPF incidence with increasing age was seen in Norway and the UK $[7,8,20]$. Higher IPF incidence was observed among males than females in the UK $[7,8]$, but not in Norway (4.0 versus 4.6 per 100,000 person-yrs for males and females, respectively) (figs 2 and 3) [20].

In the USA, IPF incidence decreased over time from 1997 to 2005 [11], while in the UK incidence increased from 1991 to 2003 [7] and during 2000-2008 (fig. 4) [8]. Interestingly, the recent IPF incidence figures in the UK (6.78 per 100,000 personyrs in 2000-2003 [7], and 7.44 per 100,000 person-yrs in 20002008 [8]) are within the range of the narrow IPF case criteria (5.96 per 100,000 person-yrs) and the broad IPF case criteria (11.04 per 100,000 person-yrs) of the US IPF incidence figures for 2003-2005. The increasing annual incidence of IPF was also reported in the Czech Republic between 1981 and 1990, from 0.74 to 1.28 cases per 100,000 population [14]. In summary, IPF incidence increases with age, peaking in patients $\geqslant 75 \mathrm{yrs}$ of

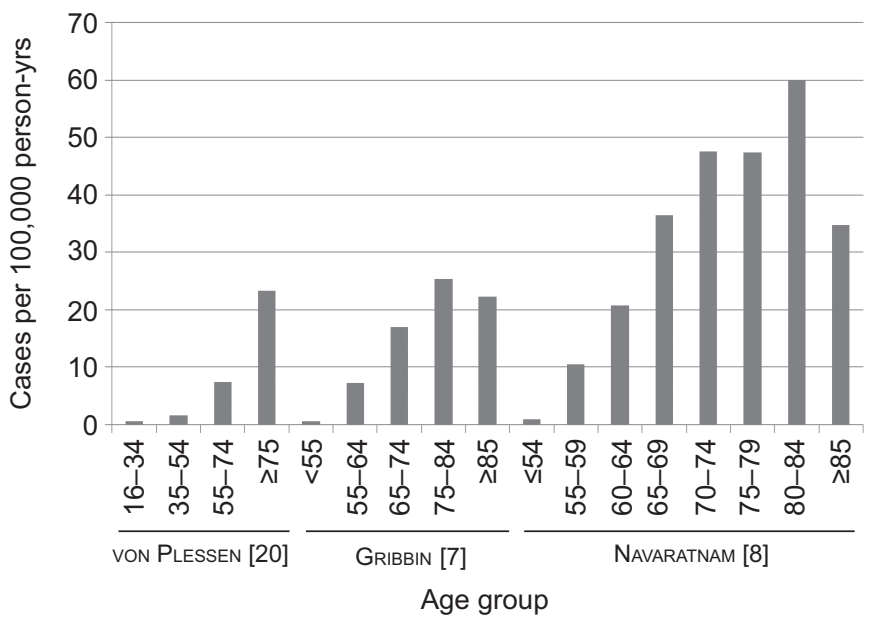

FIGURE 2. Age-stratified incidence of idiopathic pulmonary fibrosis.

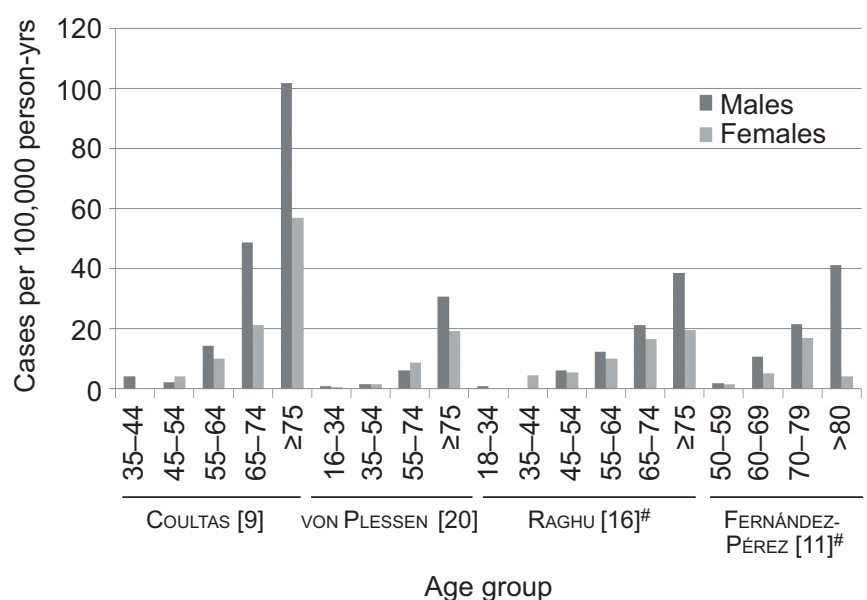

FIGURE 3. Age- and sex-stratified incidence of idiopathic pulmonary fibrosis \# : narrow case definition.

age. While studies from Europe indicate increasing IPF incidence during the observation period, data from the USA show declining incidence.

\section{Proportion of IPF among subjects with incident ILD}

Incident cases of IPF were compared across ILD registries in European countries and the USA [18]. In Belgium (Flanders), $19 \%$ of incident ILD cases were IPF, while in Germany and the USA the proportions of IPF were $32 \%$ and $31 \%$ among incident ILD cases, respectively. In Spain, XAUBET et al. [21] reported that $38.6 \%$ of incident ILD cases were attributed to IPF. Among ILD incidence diagnoses, IPF accounts for 19-39\%.

\section{DISCUSSION}

The incidence and prevalence of IPF are difficult to determine due to the lack of uniform diagnostic criteria, which were only recently established $[1,6]$. We reviewed relevant literature of the past two decades on the global prevalence and incidence of IPF. All studies reported data from North America, Europe or Japan. We did not find studies on IPF prevalence or incidence

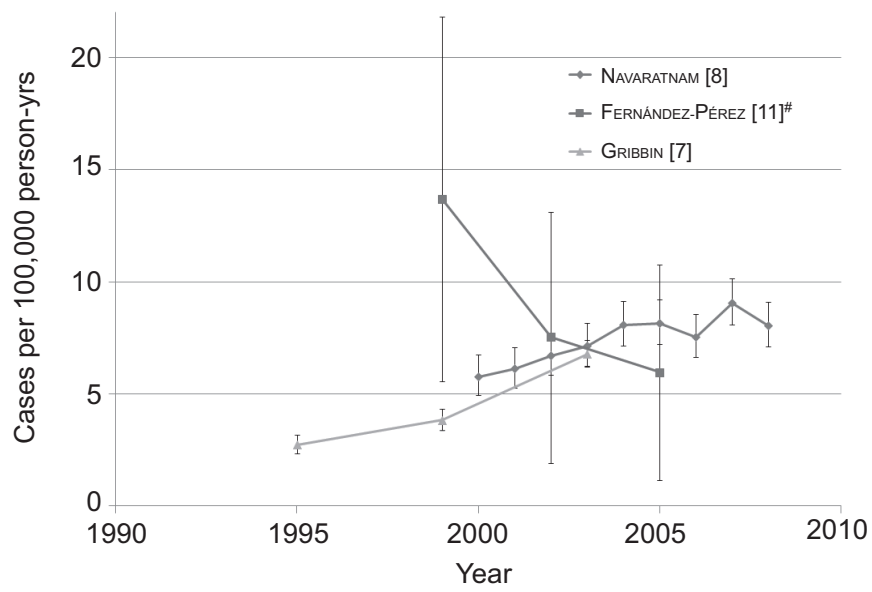

FIGURE 4. Incidence of idiopathic pulmonary fibrosis over time. The ranges of years are represented as the final year in the range, i.e. 1997-1999 is plotted under 1999. The error bars represent $95 \%$ confidence intervals. ${ }^{*}$ : narrow case definition. 
in other geographic regions (e.g. South America, Africa or Australia) published in the English language.

In the reviewed studies, prevalence varied depending on the case definition used. In the USA, prevalence estimates ranged between 14 and 27.9 cases per 100,000 of the general population using narrow case definitions, and between 42.7 and 63 cases per 100,000 population using broad case definitions. Prevalence estimates in Europe ranged from 1.25 to 23.4 per 100,000 population. The annual incidence of IPF in the USA was estimated at $6.8-8.8$ per 100,000 population using narrow case definitions and $16.3-17.4$ per 100,000 population using broad case definitions, while in Europe the annual incidence ranged from 0.22 to 7.4 per 100,000 population. Both prevalence and incidence estimates reported in the USA tended to be higher than those reported in Europe or Japan. Prevalence estimates increased with increasing age. In the USA, prevalence was higher among males than females, in contrast to a study in Norway in which females had higher prevalence rates. Prevalence in the USA also appears to be on the increase in recent years. However, reported prevalence values are still below the thresholds defining an orphan disease both under the Orphan Drug Act in the USA (a disease affecting $<200,000$ people in the USA) [23], as well as the European criteria for Orphan designation (a condition affecting no more than five out of 10,000 people in the European Union) [24]. Incidence estimates were also higher with increasing age and among males compared with females, except those reported from Norway. In the USA, it seems that the incidence of IPF decreased in recent years, while in the UK incidence reported lately is higher than that reported previously. However, the recent incidence estimates in the USA are similar to the recent incidence estimates in the UK.

The variability observed between studies is not surprising when a number of intervening elements are taken into consideration. IPF is difficult to diagnose with certainty, and diagnostic criteria have evolved in recent years. For example, IPF and non-specific interstitial pneumonia (NSIP) should be considered differential diagnoses because NSIP might have a clinical presentation similar to IPF but a more favourable prognosis and response to immunosuppresive therapy $[25,26]$. Mixed patient populations of IPF and other chronic interstitial pneumonias with different prognoses are suspected to have influenced variability in epidemiologic study results from previous reports [27]. The use of HRCT, an important diagnostic tool for IPF, has become more widely available than it was a few years ago. In fact, according to the 2011 ATS guidelines, diagnosis of IPF requires exclusion of known causes of ILD and the presence of UIP pattern on a HRCT scan in patients for whom surgical lung biopsy is not indicated [1]. Additionally, studies reporting on IPF epidemiology used neither similar case definitions (e.g. code system changes over time [8], and narrow and broad definitions $[9,11,16,20])$ nor equivalent populations. For example, all studies conducted in the USA focused on adult populations, aged $\geqslant 18$ or $\geqslant 50 \mathrm{yrs}$, while European studies provided estimates primarily for all ages; some studies were conducted using healthcare claims databases, while others used disease registries or general practice databases. Therefore, epidemiological estimates from different studies cannot be directly compared without taking their heterogeneity into consideration.
Furthermore, there seems to be certain geographic variability because there were differences across regions reported in the same study using the same methodology [8, 14]. Geographic variability may be the consequence of different diagnostic capabilities [8, 14] and also differential distribution of risk factors across regions, such as environmental exposure [28, 29], smoking habits [28, 30], certain viral infections [31], ethnic background [32] or genetics [33, 34], which may have an impact on IPF incidence [1,3]. Geographic variability of IPF prevalence may also be influenced by differences in mortality, possibly as a result of the influence of comorbidities such as pulmonary hypertension [35, 36], emphysema [35], pulmonary embolism, coronary artery disease, lung cancer or diabetes mellitus [1-3].

IPF remains a rare and challenging condition of unknown cause with an unpredictable clinical course and high mortality. Whilst still being below the thresholds defining an orphan disease, its incidence and prevalence have been increasing in most western societies; this does not appear to be associated with ageing of the population. In the UK there are potentially 15,000 patients with an IPF diagnosis, with approximately 5,000 new patients diagnosed each year [8]. In the USA there are approximately 89,000 patients with IPF and 14,000-34,000 new patients each year [16]. These patients currently have a serious unmet medical need due to IPF.

\section{SUPPORT STATEMENT}

This study was supported by Boehringer Ingelheim $\mathrm{GmbH}$.

\section{STATEMENT OF INTEREST}

L. Nalysnyk was an employee of United BioSource Corporation at the time of study submission; he is now an employee of Genzyme Corporation. Genzyme Corporation had no involvement in the study. J. Cid-Ruzafa and P. Rotella are employees of United BioSource Corporation. D. Esser is an employee of Boehringer Ingelheim.

\section{REFERENCES}

1 Raghu G, Collard HR, Egan JJ, et al. An official ATS/ERS/JRS/ ALAT statement: idiopathic pulmonary fibrosis: evidence-based guidelines for diagnosis and management. Am J Respir Crit Care Med 2011; 183: 788-824.

2 King TE Jr, Pardo A, Selman M. Idiopathic pulmonary fibrosis. Lancet 2011; 378: 1949-1961.

3 Meltzer EB, Noble PW. Idiopathic pulmonary fibrosis. Orphanet J Rare Dis 2008; 3: 8 .

4 Kim DS, Collard HR, King TE Jr. Classification and natural history of the idiopathic interstitial pneumonias. Proc Am Thorac Soc 2006; 3: 285-292.

5 Lamas DJ, Kawut SM, Bagiella E, et al. Delayed access and survival in idiopathic pulmonary fibrosis: a cohort study. Am J Respir Crit Care Med 2011; 184: 842-847.

6 Idiopathic pulmonary fibrosis: diagnosis and treatment. International consensus statement. American Thoracic Society (ATS) and the European Respiratory Society (ERS). Am J Respir Crit Care Med 2000; 161: 646-664.

7 Gribbin J, Hubbard RB, Le Jeune I, et al. Incidence and mortality of idiopathic pulmonary fibrosis and sarcoidosis in the UK. Thorax 2006; 61: 980-985.

8 Navaratnam V, Fleming KM, West J, et al. The rising incidence of idiopathic pulmonary fibrosis in the UK. Thorax 2011; 66: 462-467. 
9 Coultas DB, Zumwalt RE, Black WC, et al. The epidemiology of interstitial lung diseases. Am J Respir Crit Care Med 1994; 150: 967-972.

10 Dinwiddie R, Sharief N, Crawford O. Idiopathic interstitial pneumonitis in children: a national survey in the United Kingdom and Ireland. Pediatr Pulmonol 2002; 34: 23-29.

11 Fernández Pérez ER, Daniels CE, Schroeder DR, et al. Incidence, prevalence, and clinical course of idiopathic pulmonary fibrosis a population-based study. Chest 2010; 137: 129-137.

12 Hodgson U, Laitinen T, Tukiainen P. Nationwide prevalence of sporadic and familial idiopathic pulmonary fibrosis: evidence of founder effect among multiplex families in Finland. Thorax 2002; 57: 338-342.

13 Karakatsani A, Papakosta D, Rapti A, et al. Epidemiology of interstitial lung diseases in Greece. Respir Med 2009; 103: 1122-1129.

14 Kolek V. Epidemiology of cryptogenic fibrosing alveolitis in Moravia and Silesia, in the period 1981-1990. Internista 1995; 3: 105-108.

15 Ohno S, Nakaya T, Bando M, et al. Idiopathic pulmonary fibrosis results from a Japanese nationwide epidemiological survey using individual clinical records. Respirology 2008; 13: 926-928.

16 Raghu G, Weycker D, Edelsberg J, et al. Incidence and prevalence of idiopathic pulmonary fibrosis. Am J Respir Crit Care Med 2006; 174: 810-816.

17 Thomeer M, Demedts M, Vandeurzen K. Registration of interstitial lung diseases by 20 centres of respiratory medicine in Flanders. Acta Clin Belg 2001; 56: 163-172.

18 Thomeer MJ, Costabel U, Rizzato G, et al. Comparison of registries of interstitial lung diseases in three European countries. Eur Respir J 2001; 18: Suppl. 32, 114s-118s.

19 Tinelli C, De Silvestri A, Richeldi L, et al. The Italian register for diffuse infiltrative lung disorders (RIPID): a four-year report. Sarcoidosis Vasc Diffuse Lung Dis 2005; 22: Suppl. 1, S4-S8.

20 von Plessen C, Grinde O, Gulsvik A. Incidence and prevalence of cryptogenic fibrosing alveolitis in a Norwegian community. Respir Med 2003; 97: 428-435.

21 Xaubet A, Ancochea J, Morell F, et al. Report on the incidence of interstitial lung diseases in Spain. Sarcoidosis Vasc Diffuse Lung Dis 2004; 21: 64-70.

22 American Thoracic Society, European Respiratory Society. American Thoracic Society/European Respiratory Society International Multidisciplinary Consensus Classification of the Idiopathic Interstitial Pneumonias. Am J Respir Crit Care Med 2002; 165: 277-304.

23 U.S. Food and Drug Administration. Definition of Disease Prevalence for Therapies Qualifying under the Orphan Drug Act.
www.fda.gov/ForIndustry/DevelopingProductsforRareDiseases Conditions/HowtoapplyforOrphanProductDesignation/Definitionof DiseasePrevalence/ucm135130.htm Date last updated: July 9, 2010 Date last accessed: March 12, 2012.

24 European Medicines Agency. Orphan Designation. www.ema. europa.eu/ema/index.jsp?curl=pages/regulation/general/general_ content_000029.jsp\&mid=WC0b01ac05800240ce Date last accessed: March 12, 2012.

25 Turner-Warwick M, Burrows B, Johnson A. Cryptogenic fibrosing alveolitis: clinical features and their influence on survival. Thorax 1980; 35: 171-180.

26 Turner-Warwick M, Burrows B, Johnson A. Cryptogenic fibrosing alveolitis: response to corticosteroid treatment and its effect on survival. Thorax 1980; 35: 593-599.

27 Bjoraker JA, Ryu JH, Edwin MK, et al. Prognostic significance of histopathologic subsets in idiopathic pulmonary fibrosis. Am J Respir Crit Care Med 1998; 157: 199-203.

28 Baumgartner KB, Samet JM, Coultas DB, et al. Occupational and environmental risk factors for idiopathic pulmonary fibrosis: a multicenter case-control study. Collaborating Centers. Am J Epidemiol 2000; 152: 307-315.

29 Miyake Y, Sasaki S, Yokoyama T, et al. Occupational and environmental factors and idiopathic pulmonary fibrosis in Japan. Ann Occup Hyg 2005; 49: 259-265.

30 Steele MP, Speer MC, Loyd JE, et al. Clinical and pathologic features of familial interstitial pneumonia. Am J Respir Crit Care Med 2005; 172: 1146-1152.

31 Egan JJ, Stewart JP, Hasleton PS, et al. Epstein-Barr virus replication within pulmonary epithelial cells in cryptogenic fibrosing alveolitis. Thorax 1995; 50: 1234-1239.

32 Lederer DJ, Arcasoy SM, Barr RG, et al. Racial and ethnic disparities in idiopathic pulmonary fibrosis: a UNOS/OPTN database analysis. Am J Transplant 2006; 6: 2436-2442.

33 Lee HL, Ryu JH, Wittmer MH, et al. Familial idiopathic pulmonary fibrosis: clinical features and outcome. Chest 2005; 127: 2034-2041.

34 Rosas IO, Ren P, Avila NA, et al. Early interstitial lung disease in familial pulmonary fibrosis. Am J Respir Crit Care Med 2007; 176: 698-705.

35 Cottin V, Le Pavec J, Prevot G, et al. Pulmonary hypertension in patients with combined pulmonary fibrosis and emphysema syndrome. Eur Respir J 2010; 35: 105-111.

36 Lettieri CJ, Nathan SD, Barnett SD, et al. Prevalence and outcomes of pulmonary arterial hypertension in advanced idiopathic pulmonary fibrosis. Chest 2006; 129: 746-752. 